\title{
Structural transformations in self- assembled semiconductor quantum dots as inferred by transmission electron microscopy
}

Peter Mock, Yuanyuan Lei, Teya Topuria, Nigel D. Browning, Regina Ragan, et al.

Peter Mock, Yuanyuan Lei, Teya Topuria, Nigel D. Browning, Regina Ragan, Kyu S. Min, Harry A. Atwater, "Structural transformations in selfassembled semiconductor quantum dots as inferred by transmission electron microscopy," Proc. SPIE 4807, Physical Chemistry of Interfaces and Nanomaterials, (15 November 2002); doi: 10.1117/12.450998

SDIE Event: International Symposium on Optical Science and Technology, 2002, Seattle, WA, United States 


\title{
Structural Transformations in self-assembled Semiconductor Quantum Dots as inferred by Transmission Electron Microscopy
}

\author{
Peter Möck ${ }^{* 1}$, Yuanyuan Lei ${ }^{1}$, Teya Topuria ${ }^{1}$, Nigel D. Browning ${ }^{1}$, Regina Ragan ${ }^{* * 2}$, \\ Kyu S. Min ${ }^{* * * 2}$, and Harry A. Atwater ${ }^{2}$ \\ ${ }^{1}$ Department of Physics, MC 273, University of Illinois at Chicago, 845 W. Taylor Street, Chicago, \\ Illinois 60607-7059
}

2 Thomas J. Watson Laboratory of Applied Physics, California Institute of Technology, MS 128-95, Pasadena, CA 91125

\begin{abstract}
Transmission electron microscopy studies in both the scanning and parallel illumination mode on samples of two generic types of self-assembled semiconductor quantum dots are reported. III-V and II-VI quantum dots as grown in the Stranski-Krastanow mode are typically alloyed and compressively strained to a few \%, possess a more or less random distribution of the cations and/or anions over their respective sublattices, and have a spatially non-uniform chemical composition distribution. Sn quantum dots in $\mathrm{Si}$ as grown by temperature and growth rate modulated molecular beam epitaxy by means of two mechanisms possess the diamond structure and are compressively strained to the order of magnitude $10 \%$. These lattice mismatch strains are believed to trigger atomic rearrangements inside quantum dots of both generic types when they are stored at room temperature over time periods of a few years. The atomic rearrangements seem to result in long-range atomic order, phase separation, or phase transformations. While the results suggest that some semiconductor quantum dots may be structurally unstable and that devices based on them may fail over time, triggering and controlling structural transformations in self-assembled semiconductor quantum dots may also offer an opportunity of creating atomic arrangements that nature does not otherwise provide.
\end{abstract}

Keywords: semiconductor quantum dots, atomic ordering, phase separation, phase transformation, nanoscience

\section{INTRODUCTION}

Self-assembled semiconductor quantum dots (QDs) are expected to lead to "paradigm changes in semiconductor physics"1. As reviewed in recent textbooks ${ }^{2,3}$, improved optoelectronic devices (such as lasers ${ }^{4,5}$ and mid-infrared detectors $^{6}$ ) and novel microelectronic concepts which depend on single electron transport, tunneling, or interactions (e.g. quantum cellular automata ${ }^{7}$ ), have either been realized or may be realized as the field matures. For optoelectronic devices, the QDs need to consist of appropriately sized semiconductor entities of a smaller bandgap that are embedded in a semiconductor matrix of a larger bandgap. No structural defects (such as dislocations), which lead to non-radiative recombination centers, are allowed to exist in the QDs if the device is to function efficiently ${ }^{4}$.

Analogous to the well known particle in a box model, the simplest quantum mechanical model describes a QD as a finite potential-barrier trap for an exciton. When the size of this trap is in all three dimensions smaller than the bulk exciton Bohr radius (order of magnitude $10 \mathrm{~nm}$ ), discrete energy levels for the electron and hole arise from the spatial confinement of these particles. The energy separation of the electron levels is for (In,Ga)As of the order of magnitude $100 \mathrm{meV}^{1,8}$, i.e. significantly larger than the thermal energy at room temperature $(\mathrm{kT} \sim 26 \mathrm{meV})$. It is these discrete energy states of an electron in a QD that are exploited in devices that work at room temperature.

As in the particle in a box model, the spacing of the energy levels in QDs depends sensitively ${ }^{8}$ on a range of parameters of the matter wave trap. For a QD, these parameters are first and foremost its size, shape, net chemical

now at: * Portland State University, Department of Physics, P.O. Box 751, Portland, OR 97207-0751, peter_moeck@ hotmail.com, pmoeck@pdx.edu; ** Hewlett-Packard Laboratories, 1501 Page Mill Rd., MS 1123, Palo Alto, CA 94304; *** Intel Corporation, California Technology and Manufacturing, MS RNB-2-35, 2200 Mission College Blvd., Santa Clara, CA 95052-8119 
composition and spatial chemical composition distribution, net lattice mismatch strain and spatial strain distribution, crystallographic phase and orientation. Since a very large number of QDs (order of magnitude $10^{5}$ for vertical cavity surface emitting lasers ${ }^{4}$ and order of magnitude $10^{7}$ for edge emitting lasers ${ }^{5}$ ) are employed in laser devices, it is obvious that variations in the parameters of the individual matter wave traps are to be as small as possible in order to circumvent undue broadening of the discrete electron energy levels on which the operation of the device relies. Although addressing this uniformity issue of QDs by optimized growth and post-growth annealing processes is still a challenge, optimizations of the growth process have been achieved in recent years on the basis of structural and spectroscopic analyses.

Structural analyses of QDs are mainly performed by transmission electron microscopy (TEM) and near surface sensitive X-ray scattering techniques. Only very few of such investigations, however, address the long-term structural and morphological stability of QDs at room temperature over a time period of a few years. Such structural investigations are, therefore, to be presented in this paper for samples of two generic types of QDs and the respective morphological and structural transformations to be inferred.

The first generic type of QD we are going to deal with is currently most popular with the scientific community and results from growth in the Stranski-Krastanow mode (and its variants such as embedding sub-monolayers) by either molecular beam epitaxy (MBE) or metal organic chemical vapour deposition (MOCVD) $)^{2,3}$. This growth mode results in a compressive lattice mismatch strain in the QDs of a few \%. The nature of the Stranski-Krastanow transition in (In, Ga)As has recently been identified and it is believed that QD of other systems grow in the same basic manner, ${ }^{9,10}$. Due to the nature of this growth mode, the QDs are always alloyed, have a more or less random distribution of the mixed cations and/or anions over their respective sublattices, and a similar net chemical composition regardless if nominally pure substances or substitutional solid solutions are deposited. In addition, it is known that the predecessor islands and QDs have a spatially non-uniform chemical composition distribution with a core that is rich of the component with the smaller bandgap (and typically larger lattice constant), i.e. a spatially zonal chemical composition distribution ${ }^{8,11,12}$. Significant morphological transformations ${ }^{13}$ and changes in both the net chemical composition and spatial composition distribution ${ }^{11}$ of the predecessor island structures take place as a result of the embedding process. Expanding on our earlier results on structural ${ }^{14-17}$ and morphological ${ }^{18}$ transformations in III-V and II-VI Stranski-Krastanow grown QDs will be the subject of section 2 of this paper.

Sn QDs in Si represent a different generic type of QD, as they grow by endotaxy (i.e. crystallographically oriented growth in the bulk of a crystal rather than on its surface) following thermal treatments of predecessor layer structures that are deposited by temperature and growth rate modulated $\mathrm{MBE}^{19,20}$. Essentially pseudomorphic $\mathrm{Sn}_{\mathrm{x}} \mathrm{Si}_{1-\mathrm{x}}$ substitutional solutions with $\mathrm{x}=0.02-0.05$ can be grown by this method up to layer thicknesses of 1 to $2 \mathrm{~nm}$ at growth temperatures of 140 to $170{ }^{\circ} \mathrm{C}$ and a growth rate of $0.02 \mathrm{~nm}$ per second. Such $\mathrm{Sn}_{\mathrm{x}} \mathrm{Si}_{1-\mathrm{x}}$ layers are typically overgrown with 4 to $6 \mathrm{~nm}$ of $\mathrm{Si}$ at the same temperature and at growth rates ranging from 0.01 to $0.03 \mathrm{~nm}$ per second. The temperature is then raised to $550{ }^{\circ} \mathrm{C}$ and a capping layer with a thickness of the order of magnitude $100 \mathrm{~nm}$ is grown at a rate of $0.05 \mathrm{~nm}$ per second. By the time this growth sequence has been completed, the $\mathrm{Sn}_{\mathrm{x}} \mathrm{Si}_{1-\mathrm{x}}$ layer has undergone an in-situ thermal treatment at $550{ }^{\circ} \mathrm{C}$ for a time of the order of magnitude 30 minutes. In addition to this in-situ thermal treatment, ex-situ anneals at temperatures between 550 and $900{ }^{\circ} \mathrm{C}$ are performed for 30 minutes. For the growth of $\mathrm{Sn}_{\mathrm{x}} \mathrm{Si}_{1-\mathrm{x}} / \mathrm{Si}$ multilayer structures, the whole growth sequence is repeated several times, effectively resulting in an in-situ thermal treatment for the first $\mathrm{Sn}_{x} \mathrm{Si}_{1-\mathrm{x}}$ layer at $550{ }^{\circ} \mathrm{C}$ for a time on the order of magnitude a few hours ${ }^{19,20}$. This growth mode results in a compressive lattice mismatch strain in the QDs of the order of magnitude $10 \%$. There are some uncertainties as to the formation mechanism of Sn QDs, their crystallographic phase, and their long-term structural stability. We will deal with these issues in section 3 of this paper.

One can easily estimate ${ }^{21}$ that there is an essentially hydrostatic pressure in the 1 to $10 \mathrm{GPa}$ range $\mathrm{e}^{22}$ on both generic types of QD and an excess Gibbs free energy of approximately 0.1 to $1 \mathrm{eV}$ (i.e. >> kT at room temperature) for every atom of such a QD associated with the typically encountered lattice mismatch strains. It is, thus, not at all inconceivable that morphological and structural transformations should be possible over a long enough time even at room temperature, leading to a reduction or elimination of this excess Gibbs free energy. Failure of optoelectronic devices on the time scale of years may be explained by such structural transformations, as devices which employ less severely strained QDs have been found to possess longer lifetimes ${ }^{5}$. Growing thermodynamically unstable semiconductor QDs that are under compressive stress in the GPa range, structurally transforming these entities by means of suitable post-growth thermal treatments, and recovering these novel structures to ambient or device operating conditions may, on the other hand, become a way to realize Richard P. Feynman's 1959 vision of creating and exploiting atomic arrangements on the nanometer scale that nature does not otherwise provide ${ }^{23}$. 
Our structural analyses employed TEM in both the parallel illumination and scanning probe mode. A JEOL JEM2010F Schottky field emission STEM/TEM and a JEOL JEM-3010 TEM, equipped with a slow scan charge coupled device (CCD) camera, were used. Parallel illumination TEM utilized conventional (CTEM), diffraction contrast, selected area electron diffraction (SAED), and high-resolution (HRTEM) phase contrast imaging. Atomic resolution Z-contrast (high angle annular dark field) imaging in the scanning probe mode (STEM) proved to be especially useful for our investigations as the effects of strain fields in and around QDs and interference effects such as the formation of moiré fringes due to double diffraction are negligible.

The reasons for these advantages of Z-contrast STEM over HRTEM in this line of research are founded in the particulars of the Z-contrast image formation mechanism, which will be briefly discussed in the appendix. The appendix further contains a brief description of the so called undersampling artifact that may arise in digital recordings of atomic resolution TEM images and clarifies why we can exclude this artifact from our discussions. The reasons why we believe that there are no artifacts in our images that may be due to specimen damage caused by the probing electron beam in the electron microscopes will also be briefly discussed in the appendix.

TEM specimen preparation artifacts were excluded from our analysis by employing both ion-milling and chemical thinning to electron transparency. Only those structures that are not an artifact of either of these two physically different specimen preparation processes are likely to be present in both kinds of specimen. This is the classical empirical test of specimen preparation artifacts in $\mathrm{TEM}^{24}$.

\section{QUANTUM DOTS GROWN IN THE STRANSKI-KRASTANOW MODE}

Although there have been many investigations, there is still some controversy about the actual shape of both the islands that form the QDs when overgrown by a capping layer and of the QDs themselves. Our TEM determinations of the shape of quantum dots several years after the growth resulted for a III-V system, Fig. 1a, and a II-VI system ${ }^{25}$, in shapes that are oblate spheroids (convex lenses) which may have different diameters along $\pm[110]$ and $\pm[\overline{1} 10]^{13}$, making them in effect ellipsoids. This shape is known as a possible equilibrium shape of small precipitates that are elastically strained $^{26}$. Interestingly, of each spheroid, about one half was located above the level of the original wetting layer and the other half was located below this level ${ }^{25}$, Fig. 1a.

Since numerous scanning probe and TEM images of predecessor islands and QDs by other authors have shown rather different shapes to those oblate spheroids we observed, we assume that there are lattice mismatch strain driven morphological transformations over time. As the (In, Ga)Sb agglomerate in Fig. 1a is obviously dislocated but still possesses the shape of an oblate spheroid, even small lattice mismatch strains may have the capacity to cause morphological transformations over time.

Now, if one accepts that morphological transformation of QDs may take place over time, the conceptional barrier to accepting that structural transformations in QDs may also take place over a sufficiently long time is lowered. As a matter of fact, we observed in several systems, i.e. (In,Ga)Sb QDs in GaSb, Figs. 1b,c, In(As,Sb) QDs in InAs, Figs. 2a-c, $(\mathrm{Cd}, \mathrm{Zn}) \mathrm{Se}$ QDs in ZnSe, Fig. 3a, and (Cd,Mn,Zn)Se QDs in (Mn,Zn)Se, Figs. 3b-f that were grown by either MOCVD or MBE, structures that we believe came into being by such structural transformation ${ }^{14-17}$. A simple thermodynamic model calculation ${ }^{14}$ indeed suggests that such transformations should be possible as significant amounts of stored elastic energy can be released in the process.

Labeling the two shortest vectors in the power spectrum of Fig. 2a as a base of the reciprocal lattice of the atomically ordered $\operatorname{In}(\mathrm{As}, \mathrm{Sb}) \mathrm{QD}$ (for the sake of coming up with a notation only), the insert of this figure shows that the \pm 210 vectors of this quantum dot are almost at the same position in reciprocal space as the \pm 110 vectors of the InAs matrix. This suggests that this QD possesses a lattice mismatch strain minimizing orientation relationship with the matrix.

As the power spectra, Figs. 3e,f, show, the atomic arrangement in the large (Cd,Mn,Zn)Se agglomerate in Fig. 3d seems to be due to atomic ordering of the $\mathrm{Cd}$ and $\mathrm{Zn}$ cations in every second (220) plane (the nominal Mn content in the structure is only $10 \%$, so we neglect it for the description of the atomic ordering). Using the shortest vectors in Fig. 3e as base (for the sake of coming up with a notation only and assuming that the agglomerate belongs at least to the orthorhombic crystal system), the orientation relationship between agglomerate and matrix becomes $(020)_{(\mathrm{Cd}, \mathrm{Mn}, \mathrm{Zn}) \mathrm{Se}} \|$ $(2 \overline{2} 0)_{(\mathrm{Mn}, \mathrm{Zn}) \mathrm{Se}},(110)_{(\mathrm{Cd}, \mathrm{Mn}, \mathrm{Zn}) \mathrm{Se}} \|\left(\overline{1}_{13}\right)_{(\mathrm{Mn}, \mathrm{Zn}) \mathrm{Se}}$, and $[001]_{(\mathrm{Cd}, \mathrm{Mn}, \mathrm{Zn}) \mathrm{Se}} \|[110]_{(\mathrm{Mn}, \mathrm{Zn}) \mathrm{Se}}$ (whereby $\|$ symbolizes that both sets of reciprocal lattice vectors are [within the accuracy of the power spectra] parallel and of equal length, and that the direct lattice vectors are parallel). This orientation relationship is obviously lattice mismatch strain minimizing. 

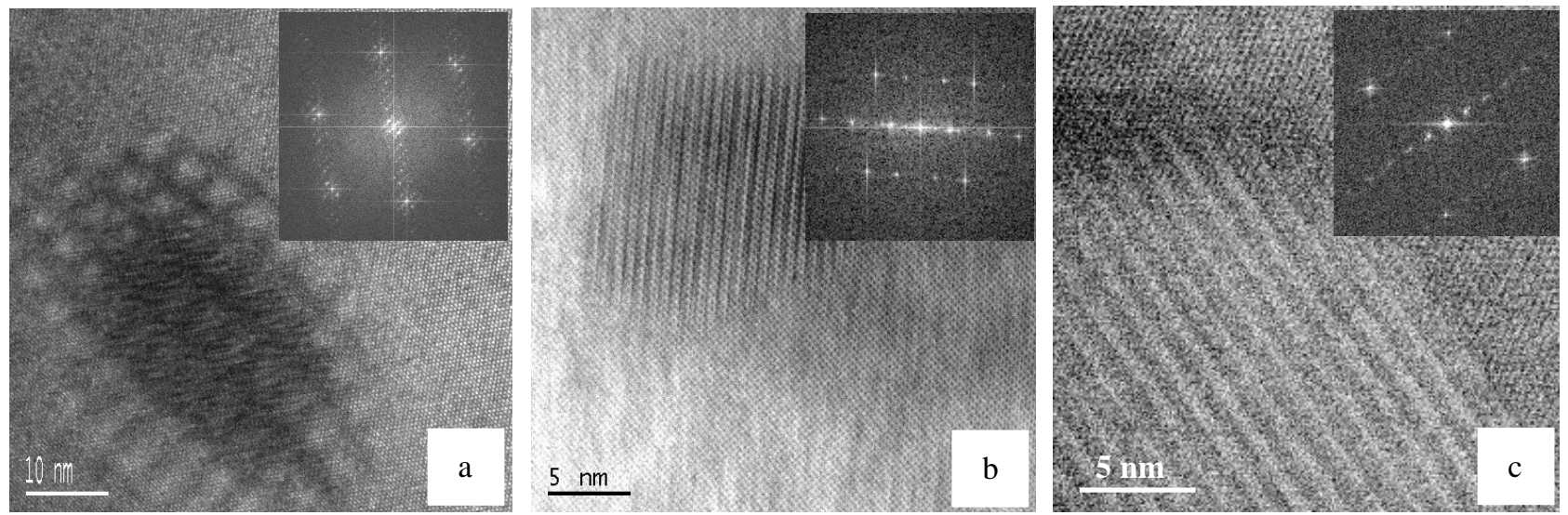

Figure 1: (In, Ga)Sb agglomerates in GaSb matrix; (a) <110> cross section HRTEM image, the 200 and 220 moiré fringes indicate that the lattice mismatch of this agglomerate is partly relaxed but the shape is nevertheless that of an oblate spheroid (convex lens); (b) [001] plan-view HRTEM image which was recorded at $500{ }^{\circ} \mathrm{C}$ and after a thermal treatment in the electron microscope at temperatures of the order of magnitude of the growth temperature for several hours, ref. 14, suggesting that the transformed structure of this QD rather than its original sphalerite prototype structure is thermodynamically stable; (c) <110> cross section Z-contrast STEM image, showing a QD with atomic ordering in every forth $\pm(002)$ plane; power spectra as inserts.
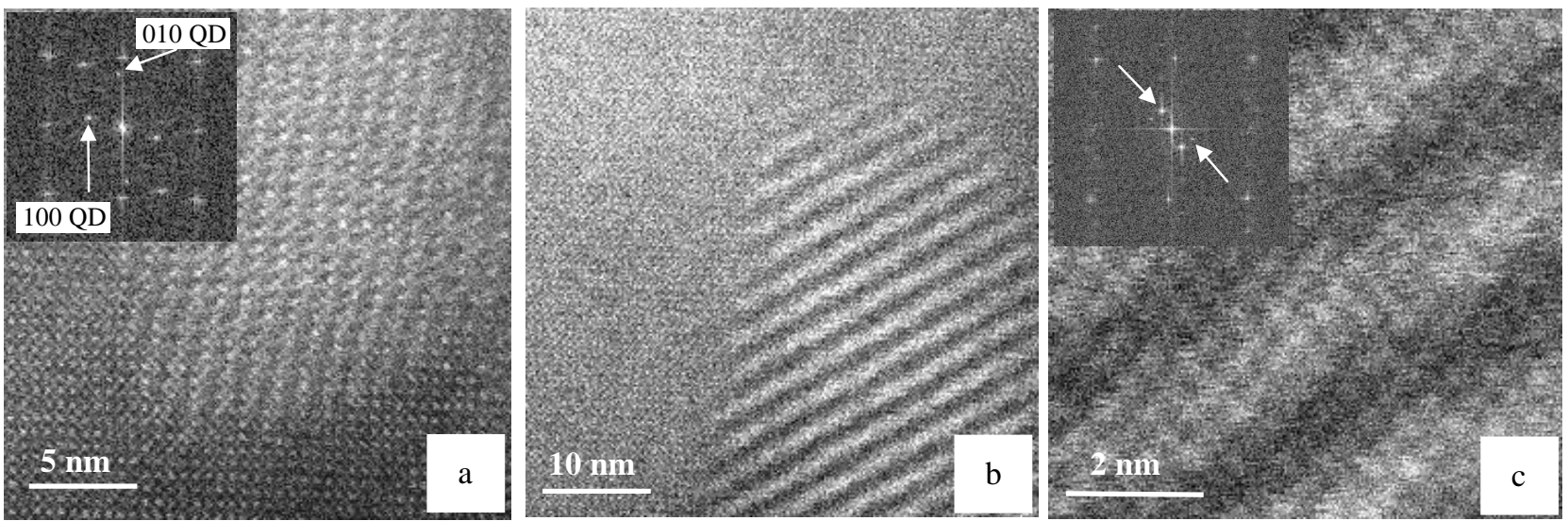

Figure 2: [001] atomic resolution Z-contrast STEM images of structurally transformed $\operatorname{In}(\mathrm{As}, \mathrm{Sb})$ QDs in InAs matrix; (a) unidentified phase that possesses a lattice mismatch strain minimizing orientation relationship with the matrix; (b) periodic compositional modulation that may have arisen from spinodal decomposition; (c) same as (b) but in higher magnification, showing atomic steps in the compositionally modulated entities that are compatible with the two observed additional periodicities in the insert power spectrum; (The power spectrum clarifies that the modulation periodicity is not due to a moiré effect since there are actually two additional periodicities, marked by arrows. These two periodicities add up to a single compositional modulation parallel to \pm [110] with a periodicity of 0.4 times the length of the vector (110) and may be explained as a lattice mismatch strain response); power spectra as inserts in (a) and (c).

Assuming that the power spectrum, Fig. 3e, is a [001] projection, the facets of the large (Cd,Mn,Zn)Se agglomerate in Fig. $3 \mathrm{~d}$ seem to be related by three mutually perpendicular two-fold axes or a two-fold axis and two mirror planes. The point symmetry group of the shape of the agglomerate may, therefore, be $222 \mathrm{or} \mathrm{mm} 2$ (requiring a relabelling in the power spectrum), but could as well be higher, i.e. $\overline{4} 2 \mathrm{~m}$ (requiring another relabelling). The point groups $222, \mathrm{~mm} 2$ and $\overline{4} 2 \mathrm{~m}$ are subgroups of $\overline{4} 3 \mathrm{~m}$. A relationship such as this would be consistent with the agglomerate resulting from atomic ordering in the sphalerite structure that corresponds to a removal of point symmetry elements from this structural prototype, e.g., removing the three-fold axis from the sphalerite structure results in the point group $\overline{4} 2 \mathrm{~m}$ [and (110), i.e. the ordering plane, is no longer symmetrically equivalent to (101) and (011)]. A shape according to either of these three point groups is also consistent with the shape of this $(\mathrm{Cd}, \mathrm{Mn}, \mathrm{Zn}) \mathrm{Se}$ agglomerate being determined by the anisotropy of the interface energy density, as determined from the application of Neumann's symmetry principle ${ }^{26,27}$.

Now, it is well known that while the shapes of small misfitting precipitates are dominated by the anisotropy of the interface energy, the shapes of larger precipitates in the same two phase systems are dominated by the anisotropy of the 
elastic mismatch energy ${ }^{26}$. The elastic mismatch energy of a precipitate is proportional to the product of the square of its lattice mismatch strain and its volume ${ }^{26}$. For the large $(\mathrm{Cd}, \mathrm{Mn}, \mathrm{Zn}) \mathrm{Se}$ agglomerate in Fig. 3d, this product is unlikely to be large, since this agglomerate probably possesses a shape that is consistent with the anisotropy of the interface energy density. As the volume of this agglomerate is, however, remarkably large, the lattice mismatch has to be minimal.

In the same specimen, there were actually several more such agglomerates which possessed the same lattice mismatch strain minimizing orientation relationship. Some of these agglomerates had diameters of up to approximately $200 \mathrm{~nm}$, and were free of structural defects such as misfit dislocations. (The agglomerate in Fig. 3d does show a lattice defect, but this is probably some kind of an antiphase boundary). We believe that it is the strain minimization effect of the particular orientation relationship which allowed the agglomerates to grow so large while retaining their translational symmetry.

As already stated above, we believe that minimization of the free energy in general and reductions or elimination of excess elastic mismatch energy in particular are the driving forces ${ }^{14}$ for the morphological and structural transformations, Figs. 1b,c, 2a-c, and 3a-d, we infer from our observations.
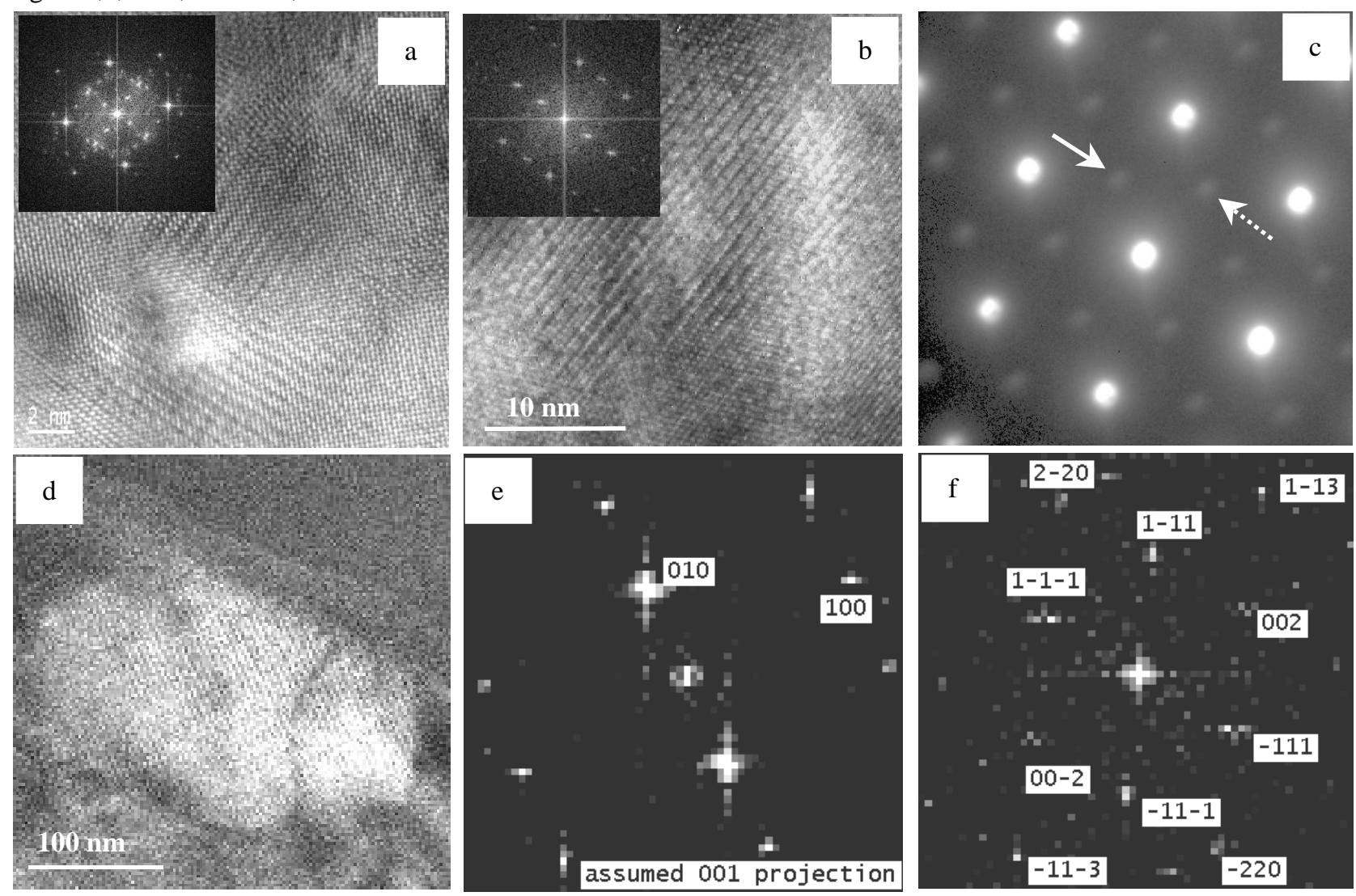

Figure 3: Atomically ordered II-VI agglomerates; (a) [001] HRTEM image, showing beginning long-range order of several modes in $(\mathrm{Cd}, \mathrm{Zn}) \mathrm{Se}$ QDs in ZnSe matrix; (b) <110> cross section HRTEM image of a (Cd,Mn,Zn)Se QD in (Mn,Zn)Se matrix, showing cation ordering in every second $\{\overline{1} 11\}$ plane, (c) $<110>$ cross section SAED pattern of (Cd,Mn,Zn)Se QDs in (Mn,Zn)Se matrix, showing ${ }^{1 / 3}$ $\{220\}$, e.g. full line arrow, and $1 / 3\{113\}$ superstructure, e.g. broken line arrow, spots; (d) $\langle 110\rangle$ cross section Z-contrast image of a large $(\mathrm{Cd}, \mathrm{Mn}, \mathrm{Zn}) \mathrm{Se}$ agglomerate in $(\mathrm{Mn}, \mathrm{Zn}) \mathrm{Se}$ matrix, note that the angels between the normals of the facets seem all to be $60^{\circ}$, indicating a rather high symmetry; (e) power spectrum of a higher magnification image of the large (Cd,Mn,Zn)Se agglomerate in (d); (f) power spectrum of a higher magnification image of the (Mn, Zn)Se matrix in (d); power spectra as inserts in (a) and (b).

\section{Sn QUANTUM DOTS IN Si}

Three sets of pairs of multilayer samples (one with and one without an additional ex-situ anneal for 30 minutes at 800 ${ }^{\circ} \mathrm{C}$ ) with four $\mathrm{Sn}_{x} \mathrm{Si}_{1-x} / \mathrm{Si}$ layers and substitutional $\mathrm{Sn}$ contents of nominally $2 \%, 5 \%$, and $10 \%$ in each of the $\mathrm{Sn}_{x} \mathrm{Si}_{1-\mathrm{x}}$ layers were grown by temperature and growth rate modulated $\mathrm{MBE}^{19,20}$, stored at room temperature for several years, and eventually selected for our TEM investigations. Fig. 4a shows such a multilayer structure in a CTEM overview. The 
$\mathrm{Sn}_{\mathrm{x}} \mathrm{Si}_{1-\mathrm{x}}$ layers appear dark in CTEM images (due to strain field influences and a combination of diffraction and absorption contrast). Most of the Sn QDs formed at or in close proximity to these layers, but there are also many Sn QDs that grew within the Si spacer layers. Figs. $4 \mathrm{~b}$ and 4c show HRTEM images of structurally defective and perfectly pseudomorphic $\mathrm{Sn}_{x} \mathrm{Si}_{1-\mathrm{x}}$ layers in $\mathrm{Si}$. The trend was, the lower the nominal Sn content in the $\mathrm{Sn}_{\mathrm{x}} \mathrm{Si}_{1-\mathrm{x}}$ layers, the lower the defect density. Samples with a nominal Sn content of $5 \%$ in these layers were found to be essentially free of such defects.

Earlier ex-situ annealing experiments at $650{ }^{\circ} \mathrm{C}$ on essentially defect free samples, Fig. 5a, indicated on the basis of plan-view TEM images an apparently very rapid increase of the average Sn QD volume $\left.(<r\rangle^{3}\right)$ with time $(t)$ for the first 2.2 hours of the anneal ${ }^{20}$. After this time, the function $\langle r\rangle^{3}(t)$ showed the typical linear behavior that is expected for precipitate coarsening by volume diffusion ${ }^{28}$. Textbook knowledge ${ }^{28}$ attributes non-linearities of $\langle\mathrm{r}\rangle^{3}$ with $\mathrm{t}$ to diffusion shortcuts such as dislocations, stacking faults, grain boundaries, and other common lattice defects.

Z-contrast STEM imaging in atomic resolution, Figs. 5b and 6a, however, revealed that within the Si matrix quite far away from the spatial positions of the $\mathrm{Sn}_{\mathrm{x}} \mathrm{Si}_{1-\mathrm{x}}$ layers (see also Fig. 4a), there are many voids in the $\mathrm{Si}$ matrix in both exsitu annealed and as grown samples (that had only had in-situ thermal treatments) which are partly filled by Sn. Fig. 6a is especially instructive as one can see that the Sn lines the interface between the void and the Si matrix. Additional evidence for the existence of voids in Si that are partly filled with $\mathrm{Sn}$ has been gathered by quantitative electron energy loss spectroscopy and will be presented elsewhere ${ }^{29}$. We consider such voids as preferential sinks for diffusing Sn atoms, i.e. as very likely candidates for the above mentioned diffusion shortcuts.

It is now important to realize that the equilibrium shape of a void in Si has been determined experimentally ${ }^{30}$ to be a tetrakaidecahedron, Fig. 6b. The applications of Neumann's symmetry principle ${ }^{26,27}$ to the determination of the shape of a small $\alpha$-Sn precipitate in a Si matrix shows that this is a tetrakaidecahedron ${ }^{31}$ as well.

Filling a void in Si with $\mathrm{Sn}$ by means of diffusion (into a diffusion shortcut) as a result of an additional thermal treatment at moderate parameters $\left(300{ }^{\circ} \mathrm{C}\right.$ for approximately three hours) directly in the electron microscope resulted in an $\alpha$-Sn QD, Fig. 5c, as $\alpha$-Sn and Si both possess the diamond structure. We consider this observation as direct proof for the void-filling hypothesis presented above. This mechanism also provides a straightforward explanation for the initially rapid increase of the average Sn QD volume with annealing time in Fig. 5a.

An interesting question is how these voids may have arisen in the first place. The possible answer to this question may be found in a mechanism analogous to that described in ref. 24, which states that when a freshly prepared Si surface is exposed to air, voids of about $10 \mathrm{~nm}$ diameter and a number density of about $10^{10} \mathrm{~cm}^{-2}$ form spontaneously approximately $10 \mathrm{~nm}$ below the surface due to compressive strain that arises from the formation of $\mathrm{SiO}_{2}$ on the surface. It does not seemed to be impossible to us that the deposited $\mathrm{Sn}_{\mathrm{x}} \mathrm{Si}_{1-\mathrm{x}}$ layers may cause the formation of voids during the growth process as well since they also compress a freshly grown Si surface. Finally, the thermal cycling during temperature and growth rate modulated $\mathrm{MBE}^{19,20}$ ensures that there is no shortage of vacancies in multilayer structures which could allow preformed voids of any shape to grow and reach their equilibrium shape and size.

Phase separation of $\mathrm{Sn}$ from a pseudomorphic $\mathrm{Sn}_{\mathrm{x}} \mathrm{Si}_{1-\mathrm{x}}$ layer will result in $\mathrm{Sn}$ (rich) QDs, Fig. 6c, since the structural prototype can be conserved. While the QDs that arise from filling of voids may consist of pure $\alpha$-Sn, the QDs that originate by phase separation are probably still substitutional solution of Sn in Si. Both types of QD will, however, be under severe lattice mismatch strains (up to $19.5 \%$ if a QD consist of pure Sn and the void is filled completely) that may make these entities unstable against structural transformations over time.

It is now very interesting that we actually observed $\beta$-Sn precipitates, Figs. 7a-c, and yet unidentified precipitates, e.g. Figs. 8a,b, as well as diffraction patterns, Fig. 8c, that suggest that there may be quite large precipitates that consist of atomically ordered Sn-Si compounds. The latter may possibly fill pits of several $10 \mathrm{~nm}$ widths and depths at the free surface (images to be shown and discussed elsewhere). While some of the $\beta$-Sn precipitates, e.g. Fig. $7 \mathrm{a}$, seemed to have associations with structural defects, other $\beta$-Sn precipitates, Fig. 7c, were observed to be surrounded by a defect free Si matrix.

For the $\beta$-Sn precipitate of Fig. 7a, the orientation relationship $(011)_{\beta-S n}\left\|(002)_{\mathrm{Si}},(600)_{\beta-S n}\right\|(\overline{4} 40)_{\mathrm{Si}}$, and $[0 \overline{1} 1]_{\beta-\mathrm{Sn}} \|$ $[110]_{\mathrm{Si}}$ can be derived from the power spectrum of this image, Fig. 7b. As this is again a lattice mismatch strain minimizing orientation relationship, this precipitate may have undergone a structural transformation from an $\alpha$-Sn QD. Such an elastic mismatch strain energy reducing structural transformation from $\alpha$-Sn may also be supported by the volume reduction that is associated with the $26 \%$ higher density of $\beta$-Sn. As $\beta-\mathrm{Sn}$ is a metal, such a structural transformation may render semiconductor devices that function on the basis of $\alpha$-Sn QD useless over time. 

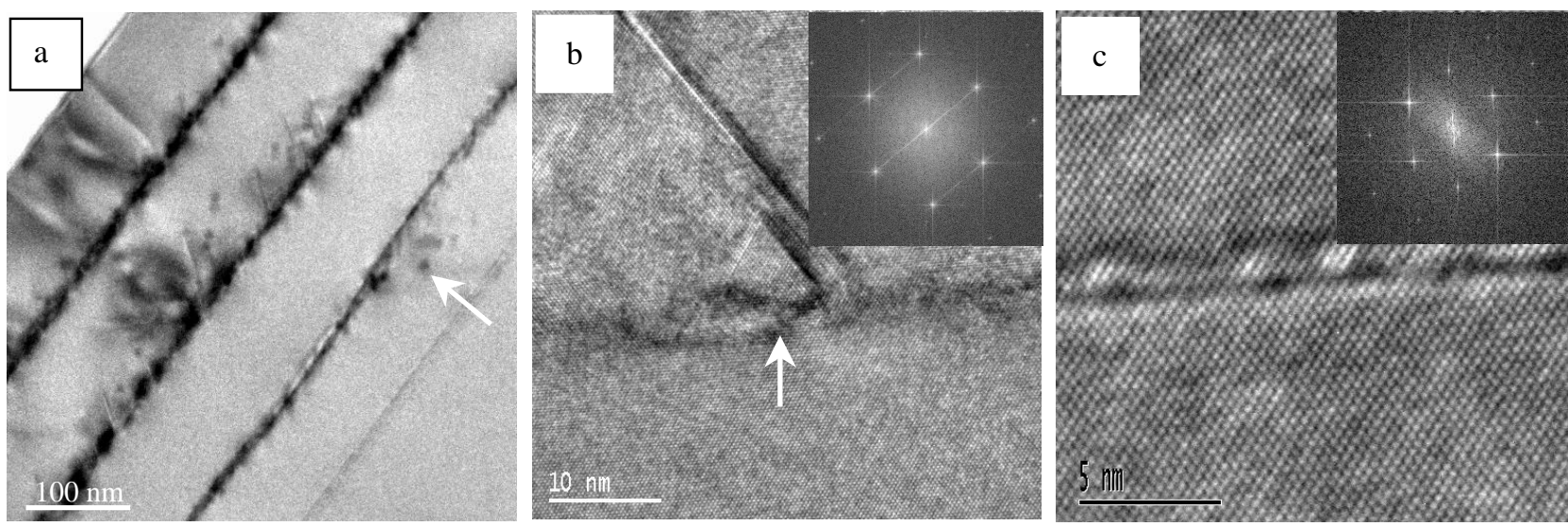

Figure 4: $\mathrm{Sn}_{\mathrm{x}} \mathrm{Si}_{1-\mathrm{x}} / \mathrm{Si}$ multilayer structures: (a) [110] cross section CTEM overview, $\mathrm{x}=0.1$, additional ex-situ anneal, the arrow is parallel to the growth direction and points towards one of many $\alpha$-Sn QDs that grew within the Si layer; (b) [110] cross section HRTEM image, $\mathrm{Sn}_{0.1} \mathrm{Si}_{0.9}$ layer (arrow) in $\mathrm{Si}$ and lattice defects on $\{1 \overline{1} 1\}$ and $\{\overline{1} 11\}$ planes, as deposited (i.e. only in-situ thermal treatments); (c) [110] cross section HRTEM image, perfectly pseudomorph $\mathrm{Sn}_{0.02} \mathrm{Si}_{0.98}$ layer in $\mathrm{Si}$, as deposited; power spectra in (b) and (c) as inserts, (arrows indicate growth directions).
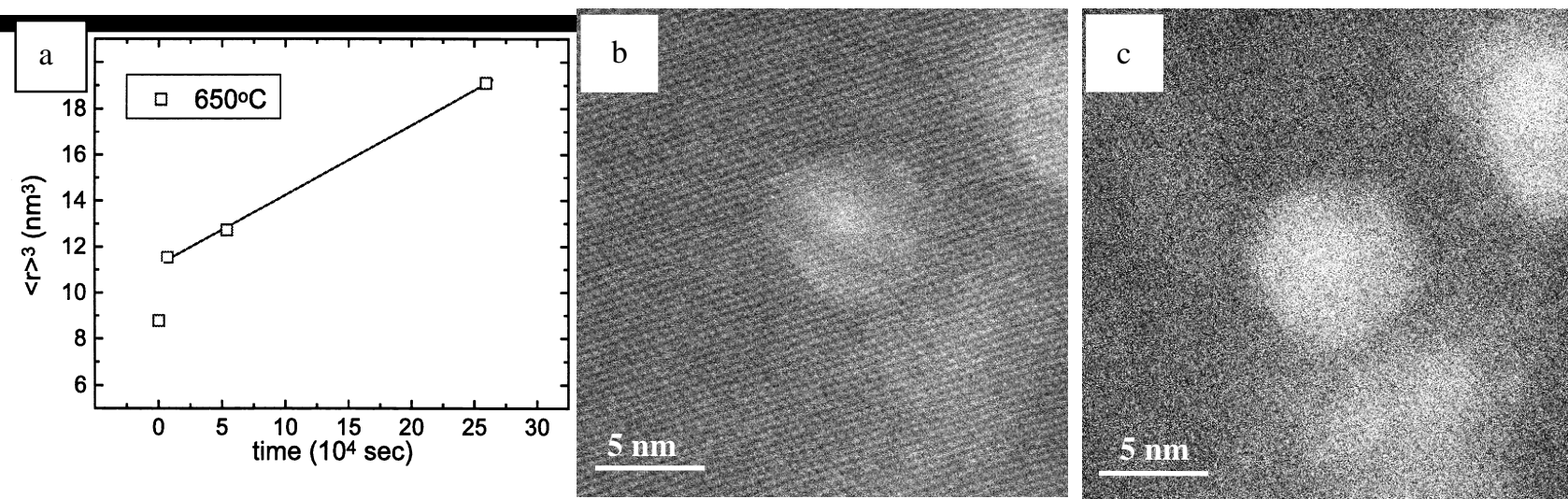

Figure 5: Diffusion shortcuts: (a) earlier results of ex-situ annealing experiments (after ref. 20); (b) [110] cross section Z-contrast STEM image of voids in Si matrix, partially filled with $\alpha$-Sn, $x=0.05$, ex-situ anneal; (c) same voids as in (b) but filled with more $\alpha$ $\mathrm{Sn}$ and grown in size as a result of a moderate additional thermal treatment inside the microscope $\left(300^{\circ} \mathrm{C}\right.$ for approximately 3 hours).
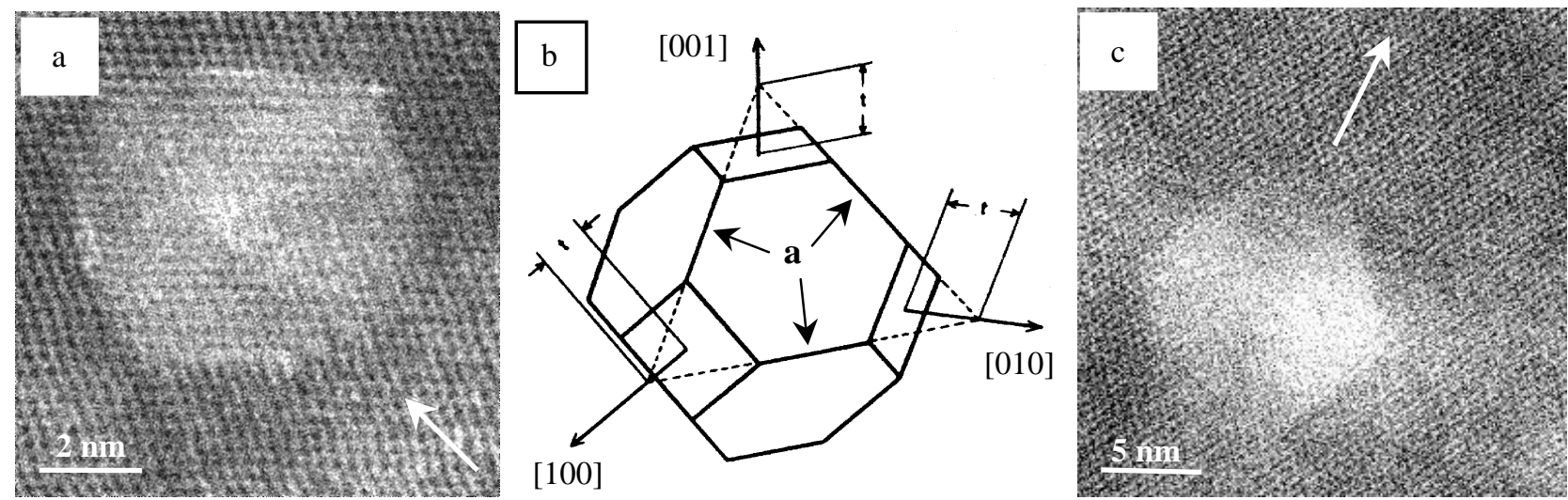

Figure 6: Tetrakaidecahedrons: (a) [110] cross section Z-contrast STEM image of a void in Si, lined on its interface with the Si matrix by $\alpha$-Sn, $\mathrm{x}=0.1$, additional ex-situ annealed; (b) sketch (after ref. 26), $\mathrm{A}=\mathrm{t} / \mathrm{a}$ is a shape parameter, $0<\mathrm{A}<2 / 3$; (c) $[110] \mathrm{cross}$ section Z-contrast STEM image of a partially formed $\mathrm{Sn}$ (rich) precipitate in $\mathrm{Si}$, grown by phase separation from a $\mathrm{Sn}_{0.1} \mathrm{Si}_{0.9}$ layer (that remains still visible as the diffuse bright band), same specimen as (a); the arrows in (a) and (c) represent the respective growth directions. 

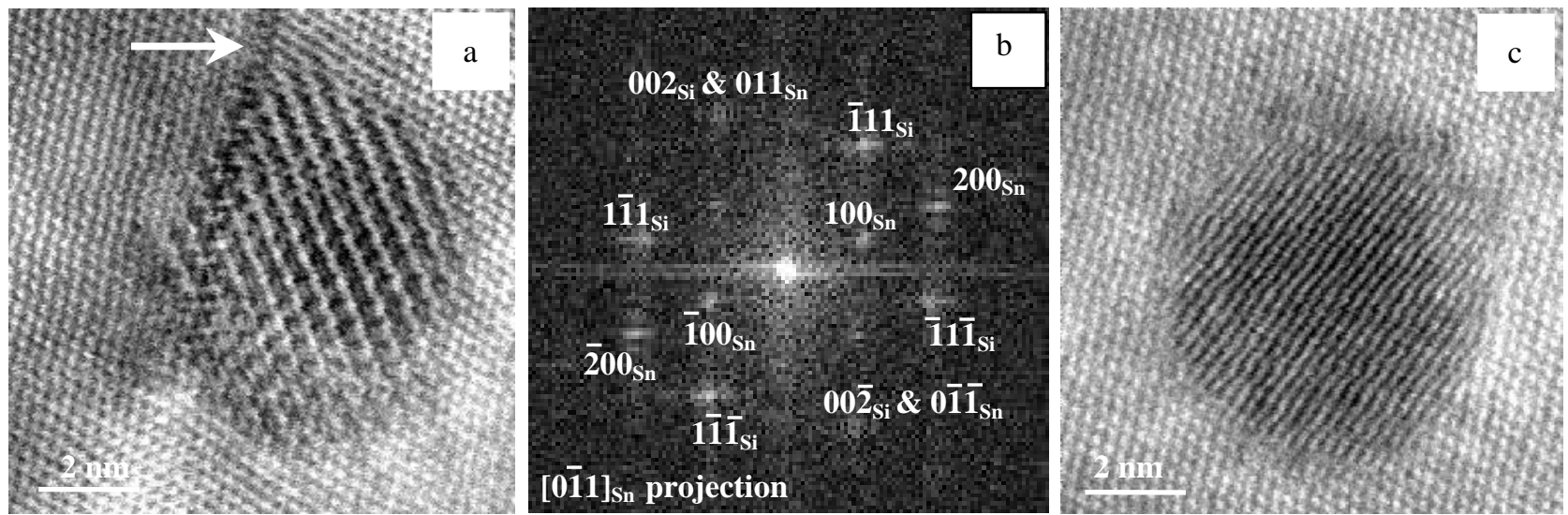

Figure 7: $\beta$-Sn precipitates in Si matrix, $x=0.1$, additional ex-situ anneal; (a) [110] cross section bright field STEM image of a precipitate that is associated with an unidentified lattice defect (arrow); (b) power spectrum (of corresponding dark field image) to (a); (c) [110] cross section bright field STEM image of a precipitate that is not associated with a lattice defect.
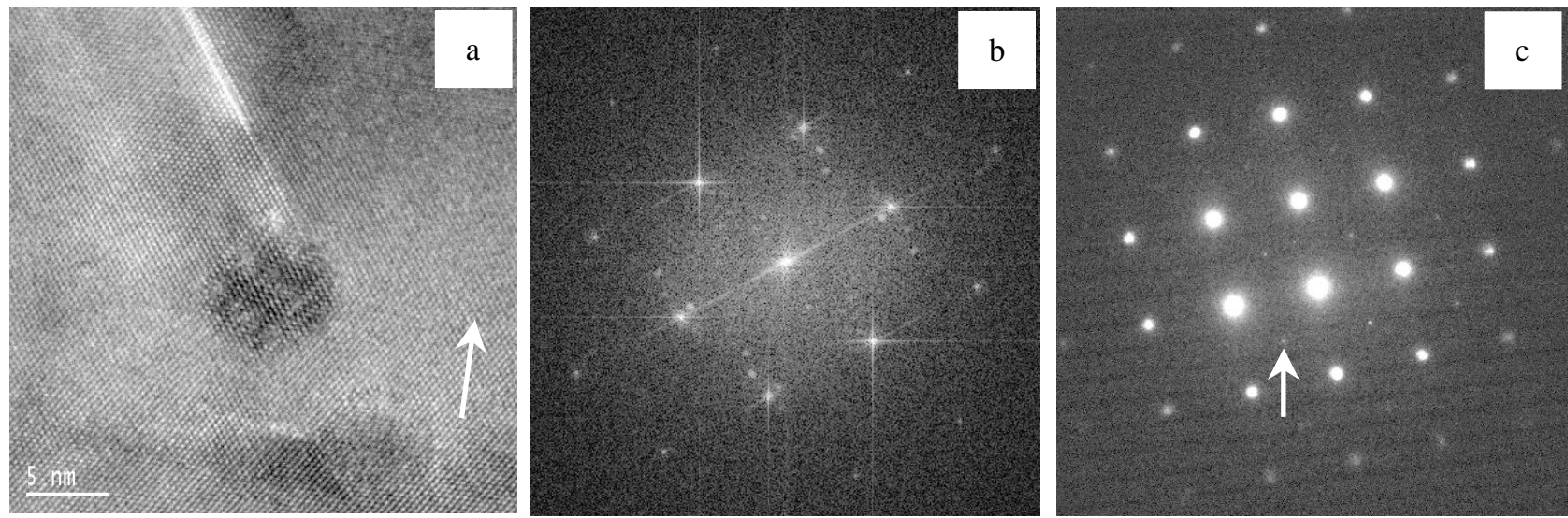

Figure 8: Other precipitates in $\mathrm{Sn}_{\mathrm{x}} \mathrm{Si}_{1-\mathrm{x}} / \mathrm{Si}$ multilayer structures; (a) [110] cross section HRTEM image of a precipitate that is associated with lattice defects on a (111) plane, $x=0.1$, additional ex-situ anneal, the growth direction is marked by the arrow; (b) power spectrum of (a), showing several unidentified periodicities; (c) [001] plan view SAED pattern, $x=0.02$, additional ex-situ anneal, showing extra spots at $1 / 2\{110\}$ (arrow) and suggesting the existence of atomically ordered Sn-Si phase precipitates.

The shape of the $\beta$-Sn precipitate of Fig. 7a is that of a rhombic-dipyramidal polyhedron, as one would expect it to be from the application of Neumann's symmetry principle ${ }^{26,27}$. Again this implies that it is the anisotropy of the interface energy density that determines the shape of this precipitate. Finally, we would like to suggest that the employment of the void creation and subsequent filling mechanism (by with $\alpha-\mathrm{Sn}$ QDs form in $\mathrm{Si}$ ) may offer an opportunity to make progress in other (less severely strained) QD systems such as InAs QDs in Si.

\section{CONCLUSIONS}

As the only common denominator of the specimens we investigated here is that several years have elapsed between the growth and the TEM analyses, the weight of the presented TEM observations (and the exclusion of possible artifacts, see appendix) in different generic types of semiconductor QDs, grown by different methods, leads us to the conclusion that there are indeed morphological and structural transformations in self-assembled QDs over time. So far, we performed only a few in-situ thermal treatments and structural analyses in the electron microscopes under elevated temperatures. More such experiments are to be performed in the future, as we expect that this will lead to irrefutable evidence for the occurrence of the structural transformations we presently only infer from our observations.

While morphological and structural transformations in QDs may turn out to be a technological problem that impacts on the lifetime of devices which function on the basis of the QDs, there is as well the great opportunity that novel semiconductor phases may be created on the nanometer scale that can only form by such transformations under pressures 
in the GPa range. In order to tackle this anticipated problem and to investigate this perceived opportunity, more TEM and other structural investigations on the above described specimens and on other samples that matured structurally over time need to be performed.

\section{APPENDIX}

Digital recordings of STEM images typically proceed in the following manner. The transmitted electrons that are scattered by the specimen into high angles are collected on an annular detector, the analog signal is digitized by an analog-digital converter and written into a data file. This data file is simultaneously displayed analog on a cathode ray tube (CRT) computer screen, Fig. 9a. It is possible to obtain Z-contrast (i.e. high angle annular dark field) images at the same time as bright field images, Fig. 9a, which are equivalent to parallel illumination TEM images by the principle of the reciprocity of ray paths and show principally the same kinds of (phase or diffraction) contrast. The magnification in a STEM image is simply the quotient of the areas that are scanned by the displaying (on the CRT) and probing (in the STEM) electron beams, whereby the display area is conveniently kept constant over a wide range of magnifications.

Detecting the scattered intensity of the transmitted electron beam at high angles and over a large angular range averages coherent effects between atomic columns in the specimen, allowing each atom to be considered to scatter independently with a cross section approaching a quadratic dependence on the atomic number ( $\mathrm{Z})$. This cross section effectively forms an object function that is strongly peaked at the atom sites. The detected intensity is a convolution of this object function with the probe intensity profile ${ }^{32}$. Fig. $9 \mathrm{~b}$, thus bypassing the phase problem that makes image interpretation difficult in HRTEM. Another important feature of this method is that changes in focus and thickness do not cause contrast reversals in the image, so that atomic sites can be identified unambiguously during the experiment ${ }^{33}$.

The small width of the object function $(\sim 0.01 \mathrm{~nm})$ means that the spatial resolution is limited only by the probe size of the microscope. For a crystalline material in a low indexed zone-axis orientation, where the atomic column spacing is greater than the probe size, the atomic columns can be illuminated individually. Therefore, as the probe is scanned over the specimen, an atomic resolution chemical compositional map is generated in which the intensity depends, in a first approximation, on the square of the average atomic number of the atoms in the column.
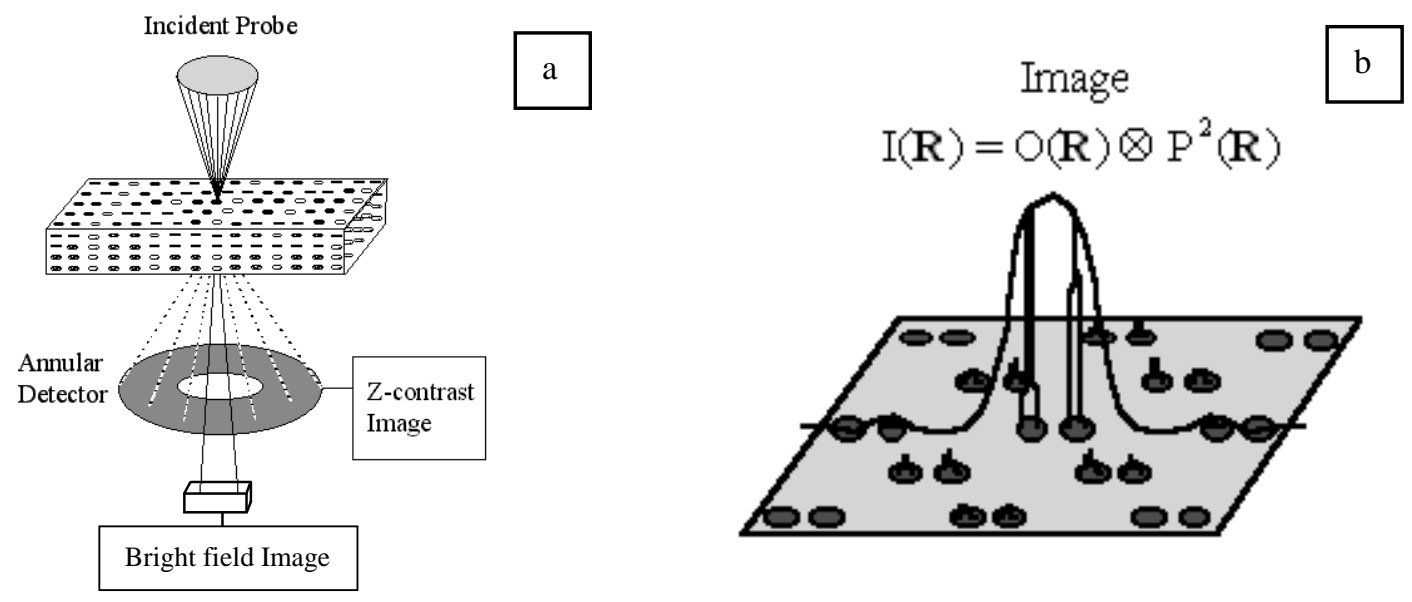

Figure 9: (a) Schematic of Z-contrast (high angle annular dark field) and bright field imaging in STEM. (b) Model to explain atomic resolution Z-contrast images; the spatial intensity $[\mathrm{I}(\mathrm{R})]$ can be interpreted as a simple convolution [ $\otimes$ ] of the experimental electron probe intensity profile $\left[\mathrm{P}^{2}(\mathrm{R})\right]$ and the object function $[\mathrm{O}(\mathrm{R})]^{32}$.

As mentioned above, due to the essentially incoherent nature of the Z-contrast image and its particular contrast mechanisms, which conceptually differs from the kinematical and dynamical theories of electron diffraction, double diffraction, and moiré fringe formation in parallel illumination $\mathrm{TEM}^{34-36}$, interference effects (such as the formation of moiré fringes) are negligible in Z-contrast images. This is demonstrated in Figs. 6a and 10a,b, where the same Sn (rich) QD is shown in both the bright field (phase contrast) and the high angle annular dark field (Z-contrast) STEM image. While the phase-contrast image, Fig. 10a, clearly shows moiré fringes, and its power spectrum, Fig. 10b, clearly shows the corresponding double diffraction spots, the Z-contrast image, Fig. 6c, only shows the compositional variation of Sn in the substitutional $\mathrm{Sn}_{\mathrm{x}} \mathrm{Si}_{1-\mathrm{x}}$ solution. 
The quality and visibility of moiré fringes in CTEM $^{34}$ and HRTEM images is known to be impeded by lattice mismatch strains, alloying and spatially inhomogeneous chemical composition distributions, i.e. effects that are all present in as grown Stranski-Krastanow QDs. It is, therefore, not surprising that moiré fringes have, to the best of our knowledge, never been observed in parallel illumination images of such QDs, the dominant contrast being due to strain fields. When atomic ordering, phase separation, and other structural transformations remove or reduce the strain fields, on the other hand, double diffraction and moiré effects become visible, as demonstrated by Figs. 1b, 3a, 4c, and 8a,b.

Artifacts that may have arisen from electron beam damage to a specimen in the electron microscope can most likely be excluded from being responsible for the TEM observations we presented in this paper. This is because, radiolytic damage mechanisms are negligible in covalent semiconductors and direct momentum transfer damage is unlikely to occur in such samples at $200 \mathrm{kV}^{36}$. In addition, different modes of atomic ordering were observed to exist in close proximity ${ }^{14}$, e.g. Fig. 10c, and it is quite incomprehensible to us how one and the same probing electron beam may have caused different modes of atomic rearrangements close to each other. On a similar token, we not only observed atomic ordering but also phase separation in one and the same specimen, Figs. 2a-c, and it is does seem unlikely that the same probing electron beam may have triggered different kinds of atomic rearrangements. Finally, we note that some of the $(\mathrm{Cd}, \mathrm{Mn}, \mathrm{Zn})$ agglomerates in $(\mathrm{Mn}, \mathrm{Zn}) \mathrm{Se}$ matrix, e.g. Fig. 3d and agglomerate no. 1 in Fig. 10c, had diameters up to approximately $200 \mathrm{~nm}$. In a sense, this seems to be by far too large to be caused by the much smaller electron beam (which has only a diameter of less than $0.2 \mathrm{~nm}$ ).
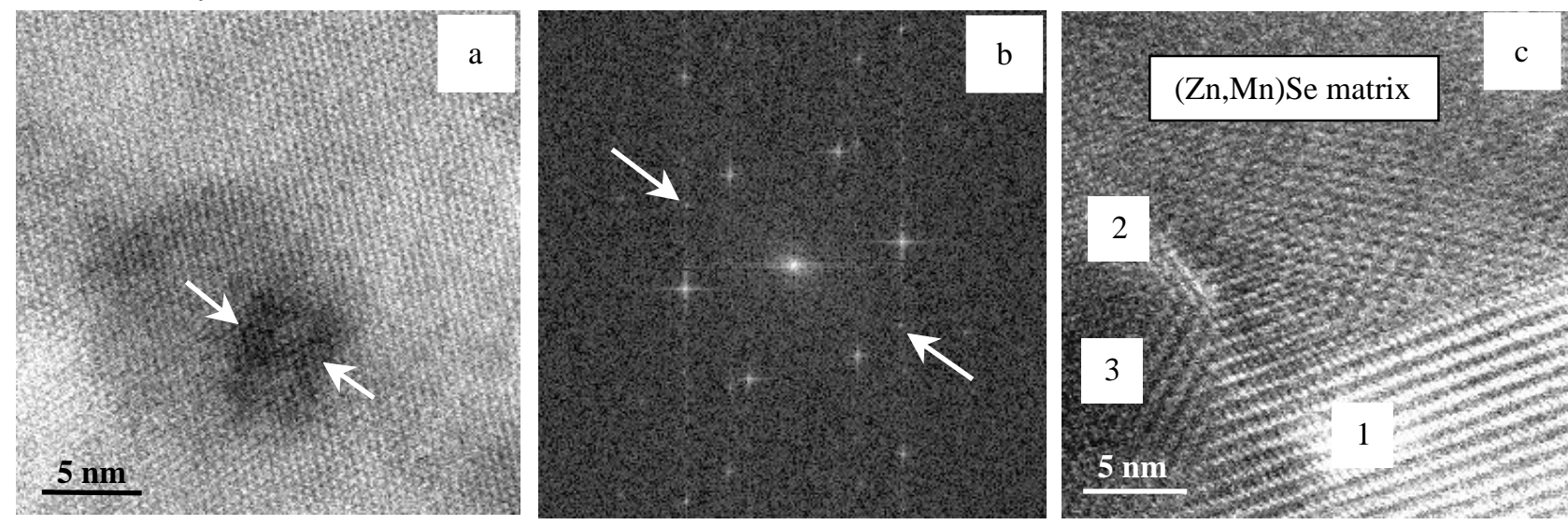

Figure 10: (a) Atomic resolution bright field STEM image complementary to Fig. 6c, showing phase contrast and two moiré fringes marked by arrows; (b) power spectrum to (a), the corresponding double diffraction spots are highlighted by arrows; (c) atomic resolution Z-contrast <110> cross section image of three different (Cd,Mn,Zn)Se agglomerates in (Mn,Zn)Se matrix.

Digitizing analog images of periodic objects such as crystals in a low index zone axis orientation at moderately high magnifications in a parallel manner by means of a CCD camera in HRTEM or in a serial manner by means of an analogdigital converter in STEM can under certain conditions cause an artifact that is called undersampling. The sampling theorem $^{12,37}$ (Nyquist-Shannon theorem) applied to CCD camera recording of atomic resolution TEM images states that both of the two smallest periodicities in the object which are to be imaged should be sufficiently magnified so that they each correspond to at least twice the width of the individual detector pixels of the CCD camera. Allowing for the arbitrary orientation of the object periodicities and the CCD camera pixels, the effective individual detector pixel width may increase by up to a factor of $\sqrt{2}$ for square pixels. When such conditions are fulfilled, no undersampling can occur. Correspondingly, avoiding undersampling in atomic resolution STEM requires the magnification to be high enough that each of the two smallest periodicities in the object is sampled at least twice by the analog-digital converter.

From the practical viewpoint, avoiding undersampling of atomic resolution STEM images requires the electron microscopist to make sure that the microscope's magnification is sufficiently large so that further increases of the magnification do not result in noticeable rotations of the image. This corresponds to the condition that no qualitative changes (other than the appropriate linear increases) occur in the power spectra of the recorded images with further increases of the magnification. As there are in most TEMs image rotations associated with increases of the magnification, the electron microscopist has to make sure in this case that the power spectra of HRTEM images do not change qualitatively either (other than the appropriate linear increases and rigid rotations). In any case, it is good practice to record/digitize images of well-known reference structures (such as the sphalerite or diamond matrices in our cases) at 
different magnifications in addition to the images of interest and check the power spectra of the former for consistency with the reference structure at all employed magnifications.

\section{ACKNOWLEDGMENTS}

Prior collaborations with G. Roger Booker, Robin J. Nicholas, (both University of Oxford) and Nigel J. Mason (Kamelian Ltd. Oxford) as well as the supply of samples by Jacek K. Furdyna, and Malgorzata Dobrowolska (both University of Notre Dame) are kindly acknowledged. Alan Nicholls (Electron Microscopy Service, University of Illinois at Chicago) is thanked for experimental support. This research was sponsored by both a grant to NDB by the National Science Foundation (DMR-9733895) and a grant to PM by the Campus Research Board of the University of Illinois at Chicago.

\section{REFERENCES}

1. D. Bimberg, "Quantum dots: Paradigm changes in semiconductor physics", Semiconductors 33, pp 951-955, 1999.

2. D. Bimberg, M. Grundmann, and N.N. Ledentsov, Quantum Dot Heterostructures, John Wiley \& Sons, Chichester, New York, Weinheim, Brisbane, Singapore, Toronto, 1999.

3. T.P. Pearsall (editor), Quantum Semiconductor Devices and Technologies, Kluwer Academic Publishers, Boston, Dordrecht, London, 2000.

4. N.N. Ledentsov, V.M. Ustinov, V.A. Shchukin, P.S. Kop'ev, Zh.I. Alferov, and D. Bimberg, "Quantum dot heterostructures: fabrication, properties, lasers (Review)", Semiconductors 32, pp 343-365, 1998.

5. H-Y. Liu, B. Xu, Y-Q. Wei, D. Ding, Q. Han, J-B. Liang, and Z-G. Wang, "High-power and long-lifetime InAs/GaAs quantum-dot laser at 1080 nm", Appl. Phys. Lett. 79, pp 2868-2870, 2001.

6. E. Towe and D. Pan, "Semiconductor quantum-dot nanostructures: Their application in a new class of infrared photodetectors", IEEE Journal of Selected Topics in Quantum Electronics 6, pp 408-421, 2000.

7. G. Snider, A.O. Orlov, R.K. Kummamuru, R. Ramasubramaniam, I. Amlani, G.H. Bernstein, and C.S. Lent, "Quantum-dot Cellular Automata", Mat. Res. Soc. Symp. Proc. Vol. 696, pp N7.6.1-N7.6.11, 2002.

8. J. Shumway, A.J. Williamson, A. Zunger, A. Passaseo, M. DeGiorgi, R. Cingolani, M. Catalano, and P. Crozier, "Electronic structure consequences of $\mathrm{In} / \mathrm{Ga}$ composition variations in self-assembled $\operatorname{In}_{\mathrm{x}} \mathrm{Ga}_{1-\mathrm{x}} \mathrm{As} / \mathrm{GaAs}$ alloy quantum dots", Phys. Rev. B 64, pp 125302-1-125302-11, 2001.

9. T. Walther, A.G. Cullis, D.J. Norris, and M. Hopkinson, "Nature of the Stranski-Krastanow Transition during Epitaxy of InGaAs on GaAs", Phys. Rev. Lett. 86, pp 2381-2384, 2001.

10. A.G. Cullis, D.J. Norris, T. Walther, M.A. Migliorato, and M. Hopkinson, "Epitaxial island growth and the StranskiKrastanow Transition', Mat. Res. Soc. Symp. Proc. Vol. 696, pp N1.1.1-N1.1.8, 2002.

11. A. Rosenauer. W. Oberst, D. Litvinov, D. Gerthsen, A. Förster, and R. Schmidt, "Structural and chemical investigation of $\mathrm{In}_{0.6} \mathrm{Ga}_{0.4} \mathrm{As}$ Stranski-Krastanow layers buried in GaAs by transmission electron microscopy", Phys. Rev. B 61, pp 8276-8288, 2000.

12. S. Kret, P. Ruterana, A. Rosenauer, and D. Gerthsen, "Extracting Quantitative Information from High Resolution Electron Microscopy", phys. stat. sol. (b) 227, pp 247-295, 2001.

13. J.M. García, G. Medeiros-Ribeiro, K. Schmidt, T. Ngo, J.L. Feng, A. Lorke, J. Kotthaus, and P.M. Petroff, "Intermixing and shape changes during the formation of InAs self-assembled quantum dots", Appl. Phys. Lett. 71, pp 2014-2016, 1997.

14. P. Möck, T. Topuria, N.D. Browning, R.J. Nicholas, G.R. Booker, “Atomic Self-ordering in Heteroepitaxially Grown Semiconductor Quantum Dots due to Relaxation of External Lattice Mismatch Strains", Mat. Res. Soc. Symp. Proc. 696, pp N8.8.1-N8.8.6, 2001.

15. P. Möck, T. Topuria, N.D. Browning, M. Dobrowolska, S. Lee, J.K. Furdyna, G.R. Booker, N.J. Mason, and R.J. Nicholas, "Internal self-ordering in $\operatorname{In}(\mathrm{Sb}, \mathrm{As}),(\mathrm{In}, \mathrm{Ga}) \mathrm{Sb}$, and (Cd,Zn,Mn)Se nano-agglomerates/quantum dots", Appl. Phys. Lett. 79, pp 946-948, 2001.

16. P. Möck, T. Topuria, N.D. Browning, G.R. Booker, N.J. Mason, R.J. Nicholas, L.V. Titova, M. Dobrowolska, S. Lee, and J.K. Furdyna, "Self-ordering in $\mathrm{CdSe} / \mathrm{ZnSe}, \mathrm{CdSe} /(\mathrm{Zn}, \mathrm{Mn}) \mathrm{Se}, \mathrm{InSb} / \mathrm{GaSb}$ and $\mathrm{InSb} / \mathrm{InAs}$ quantum dot structures and a novel type of quantum dot", Mater. Res. Soc. Symp. 640, pp P6.3.1-P.6.3.6, 2000.

17. P. Möck, T. Topuria, N.D. Browning, L. Titova, M. Dobrowolska, S. Lee, and J.K. Furdyna, "Self-Ordered CdSe Quantum Dots in ZnSe and (Zn,Mn)Se Matrices Assessed by Transmission Electron Microscopy and 
Photoluminescence Spectroscopy", J. Electron Mater. 30, pp 748-755, 2001.

18. P. Möck, G.R. Booker, N.J. Mason, R.J. Nicholas, E. Alphandéry, T. Topuria, and N.D. Browning, "MOVPE grown self-assembled and self-ordered InSb quantum dots in a GaSb matrix assessed by AFM, CTEM, HRTEM and PL", Mater. Sci. Engin. B 80, pp 112-115, 2001.

19. K.S. Min and H.A. Atwater, "Ultrathin pseudomorphic $\mathrm{Sn} / \mathrm{Si}$ and $\mathrm{Sn}_{\mathrm{x}} \mathrm{S}_{1-\mathrm{x}} / \mathrm{Si}$ heterostructures", Appl. Phys. Lett. 72, pp. 1884-1886, 1998.

20. R. Ragan, K.S. Min, and H.A. Atwater, "Direct energy gap group IV semiconductor alloys and quantum dot arrays in $\mathrm{Sn}_{\mathrm{x}} \mathrm{Ge}_{1-\mathrm{x}} / \mathrm{Ge}$ and $\mathrm{Sn}_{\mathrm{x}} \mathrm{Si}_{1-\mathrm{x}} / \mathrm{Si}$ alloy systems", Mater. Sci. Engin. B 87, 204-213, 2001.

21. For hydrostatic pressure, the product of the bulk modulus (order of magnitude $100 \mathrm{GPa}$ for semiconductors) and the relative elastic volume change is equal to the product of pressure and volume, which is also the excess Gibbs free energy due to lattice mismatch strains. Starting with an unstrained sphere of $10 \mathrm{~nm}$ diameter with a lattice constant $0.5 \mathrm{~nm}$ that contains 33510 atoms, one obtains for an relative elastic volume change of $5 \%$ a pressure of $5 \mathrm{GPa}$ and an excess Gibbs free energy of $0.49 \mathrm{eV}$ per atom. The usage of this simple formula is justified by studies of the size dependency of elastic properties of nanometer sized particles, e.g., S.P. Baker, R.P. Vinci, and T. Arias, "Elastic and Anelastic Behavior of Materials in Small Dimensions", MRS Bulletin 27, No. 1, pp 26-29, 2002 and R.E. Miller and V.B. Shenoy, "Size-dependent elastic properties of nanosized structural elements", Nanotechnology 11, pp 139-147, 2000.

22. This hydrostatic pressure range is known to lead to structural transformations in elemental (group IV) and binary III-V compound semiconductors; see, e.g., G.J. Ackland, "High-pressure phases of group IV and III-V semiconductors", Rep. Prog. Phys. 64, pp 483-516, 2001.

23. R.P. Feynman, "There is Plenty of Room at the Bottom", Lecture at the Annual Meeting of the American Physical Society, December 29, 1959, http://www.zyvex.com/nanotech/feynman.html.

24. S. Lin, I. Mack, N. Pongkrapan, and P. Fraundorf, "Ten-nanometer surface intrusions in room temperature silicon", Electrochem. Solid-State Sci. Lett. (2002), in press; http://xxx.lanl.gov/abs/cond-mat/0110393.

25. C.S. Kim, M. Kim, S. Lee, J.K. Furdyna, M. Dobrowolska, H. Rho, L.M. Smith, H.E. Jackson, E.M. James, Y. Xin and N.D. Browning, "2-D precursors and interdiffusion in CdSe/ZnSe SAQD from STEM and optical results", Phys. Rev. Lett. 85, pp 1124-1127, 2000.

26. W.C. Johnson, "Influence of Elastic Stress on Phase Transformations", in: Lectures on the Theory of Phase Transformations, 2nd Edition, Ed. H.I. Aaronson, The Minerals, Metals \& Materials Society, Warrendale, 2001.

27. As the pressure on the precipitates is in a first approximation hydrostatic, i.e. isotropic, Curie's symmetry principle, e.g. ref. 26, yields no influence of the misfit stress field on the anisotropy of the interface energy density.

28. D.A. Porter and K.E. Easterling, Phase Transformations in Metals and Alloys, Chapman \& Hall, London, New York, 1992.

29. Y. Lei, P. Möck, T. Topuria, N.D. Browning, R. Ragan, K.S. Min, and H.A. Atwater, submitted to Phys. Rev. Lett.

30. D.J. Eaglesham, A.E. White, L.C. Feldman, N. Moriya, and D.C. Jacobson, "Equilibrium Shape of Si", Phys. Rev. Lett. 70, pp 1643-1646, 1993.

31. Consistent with textbook knowledge, e.g. ref. 26, on shape transitions with size due to an increasing contribution of the elastic mismatch energy, a much larger $\alpha$-Sn precipitate $(\sim 30 \mathrm{~nm}$ radius in the [001] direction) had a shape that resembled an octahedron more closely. Intermediately sized $\alpha$-Sn precipitates showed shapes of tetrakaidecahedrons with very small $\{001\}$ facets, i.e. transitional shapes that are much closer to an octahedron than to a cube. See also P. Möck, Y. Lei, T. Topuria, N.D. Browning, R. Ragan, K.S. Min, and H.A. Atwater, "Formation mechanisms of quantum dots in the Sn/Si system", Proc. $2^{\text {nd }}$ Intern. Workshop on Quantum Nanostructures \& Nanoelectronics, AIST-Tsukuba Research Center, Tsukuba, Japan, September 9 - 11, 2002.

32. S.J. Pennycook and D.E. Jesson, "High-Resolution Incoherent Imaging of Crystals", Phys. Rev. Lett. 64, pp 938-941, 1990.

33. N.D. Browning, I. Arslan, P. Moeck and T. Topuria, "Atomic resolution STEM", phys. stat. sol. (b) 227, pp 229-245, 2001.

34. G.A. Bassett, J.W. Menter, and D.W. Pashley, "Moiré patterns on electron micrographs, and their application to the study of dislocations in metals", Proc. Royal. Soc. A 246, pp 345-368, 1958.

35. R. Gevers, "Dynamical Theory of Moire Fringe Patterns", Phil. Mag. 7, pp 1681-1720, 1962.

36. L. Reimer, Transmission Electron Microscopy, Physics of Image Formation and Microanalysis, Springer-Verlag, Berlin, Heidelberg, New York, 1989.

37. B. Jähne, Digital Image Processing, Springer-Verlag, Berlin, Heidelberg, New York, 1997. 